\title{
Performance Analysis for MRI Denoising using Intensity Averaging Gaussian Blur Concept and its Comparison with Wavelet Transform Method
}

\author{
Ami Vibhakar \\ PG student, \\ SSSIST, sehore,India
}

\author{
Mukesh Tiwari \\ Assistant professor,EC dept. \\ SSSIST, sehore, India
}

\author{
Jaikaran Singh \\ Assistant professor,EC dept. \\ SSSIST, sehore,India
}

\begin{abstract}
Feature extraction and object recognition plays important role in medical image, acquired by various imaging modalities. Medical image is taken by various technologies like MRI machine, computed tomography (CT), positron emission tomography (PET) etc. These techniques always add noise in image. Which should be removed for feature extraction and object recognition of medical image? This noise will change the phase and amplitude of every pixel in the image. So image restoration becomes essential task for perfect diagnostics of body. This noise can be reduced if phase error at each pixel in complexes valued image is known, resulting in improved detection of medically significant detail. But estimation of phase error at each pixel is difficult task. There are so many approaches, which have been previously suggested for image restoration. Here we are comparing the code wavelet method with intensity averaging Gaussian blur method and we will prove that later method is giving good result. Here we are applying no. of iterations to modify the estimate of phase error at pixel, until output converges to a stable estimate. We are using image diffusion and anisotropic method to estimate phase error at each pixel and convolution and Gaussian blurring method for amplitude correction. We are using the PSNR(Peak signal to noise ration),MSE(Mean square error) and RMSE(Root mean square error) as performance matrices to measure the quality of denoised MRI .The final result shows that this method is effectively removing the noise while preserving the edge and fine information in the images.
\end{abstract}

\section{Keywords}

MRI, Random noise, iteration, Gaussian blur, convolution, PSNR, MSE, Image denoising

\section{INTRODUCTION}

Image denoising is a word in digital image processing, focuses on the removal of noise, which may disturb an image during its acquisition or transmission. MRI is most common tool for diagnosis in Medical field. These images are mostly corrupted by random noise. The main sources of noise are RF field generated from the electronics circuit and patient. The presence of noise degrades the visual quality of image and lowers the visibility of low contrast objects. So image denoising is very important application in medical imaging applications in order to enhance and recover fine details that may be hidden in the data. MR images are typically corrupted with noise, which hinder the medical diagnosis based on these images. The process of noise suppression must not appreciably degrade the useful features in an image. In particular, edges are important features for MR images and thus the denoising must be balanced with edge preservation.
MRI is a way of getting pictures of various parts of your body without the use of x-rays, unlike regular x-rays pictures and CAT scans. A MRI scanner consists of a large and very strong magnet in which the patient lies. A radio wave antenna is used to send signals to the body and then receive signals back. These returning signals are converted into pictures by a computer attached to the scanner. Pictures of almost any part of your body can be obtained at almost any particular angle.Medical information, acquired from MRI and composed of clinical data, images and other physiological signals, has become an essential part of a patient's care for diagnosis in medical field. Over the past three decades, there is a vast development in information technology (IT) \& Medical Instrumentation ,which has improved the level of medical imaging. This development are Computed Tomography (CT), Magnetic Resonance Imaging(MRI), the different digital radiological processes for vascular, cardiovascular and contrast imaging, mammography, diagnostic ultrasound imaging, nuclear medical imaging with Single Photon Emission Computed Tomography (SPECT) and Positron Emission Tomography (PET). All these methods generate good quality of medical image [1] and each has its own specific features corresponding to the physical and physiological phenomena studied, as shown in "Fig.1
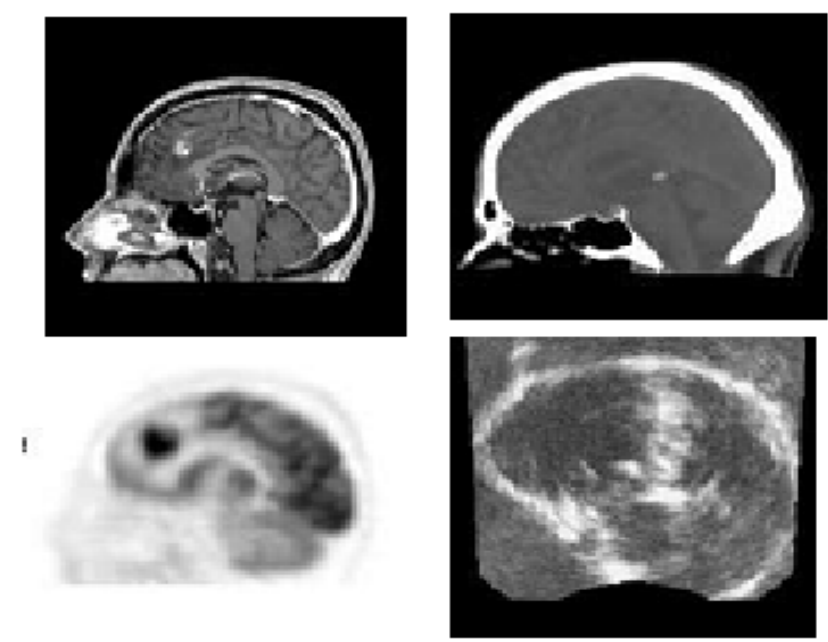

Figure1: Sagittal slices of the brain by different imaging modalities: a) magnetic resonance imaging (MRI), b) computed tomography $(\mathrm{CT}), \mathrm{c})$ positron emission tomography (PET), d) ultrasound (US) 
This paper is divided in seven sections. Section one gives idea about MRI and denoising. Section two shows a literature survey .Section three defined implementation of algorithm .Section four and five gives idea about convolution and Gaussian blur. Section six defines proposed algorithm for denoising while section seven gives conclusion

\section{RELATED WORK}

Different algorithms for image denoising are discussed in [2]. The using wave atom shrinkage methodology for de-noising of Magnetic Resonance Images is proposed in [3] and also proved that this method fives a better SNR compared to wavelet and curvelet shrinkages. Rician noise can be reduced by a NL-Denoising method, proposed in [4 \& 5].In [6],Total Variation Wavelet-Based technique is used to remove a nose from MR image. Image quality can be improved by adaptive threshold based on contourlet transform is given in [7]. A new filter to reduce random noise in multicomponent MR images by spatially averaging similar pixels and a local principal component analysis decomposition using information from all available image components to perform the denoising process is proposed in [8]. ML estimator based approach in which an estimator using a priori information for devising a single dimensional noise cancellation for the variance of the thermal noise in magnetic resonance imaging (MRI) systems has been proposed in [9]. A noise removal technique using 4th order PDE is introduced in [10] to reduce noise in MRI images.. A phase error estimation scheme based on iteratively applying a series of non-linear filters each used to modify the estimate into greater agreement with one piece of knowledge, until the output converges to a stable estimate is introduced in [11].

\section{WAVELET TRANSFORM}

In mathematics, a wavelet series is a representation of a square-integral (real- or complex-valued) function by a certain orthonormal series generated by a wavelet. This article provides a formal, mathematical definition of an orthonormal wavelet and of the integral wavelet transform. The technique works in the following way. When you decompose a data set using wavelets, you use filters that act as averaging filters and others that produce details (18). Some of the resulting wavelet coefficients correspond to details in the data set. If the details are small, they might be omitted without substantially affecting the main features of the data set. The idea of thresholding, then, is to set to zero all coefficients that are less than a particular threshold. These coefficients are used in an inverse wavelet transformation to reconstruct the data set.. The technique is a significant step forward in handling noisy image because the denoising is carried out without smoothing out the sharp structures. The result is cleaned-up image that still shows important details. Wavelet transform method performs the following steps for image denoising. Figure (A) displays an image, and then several close-up images of eye: an original, an image with noise added, and finally denoised image. To denoise the image,

[1] Transformed the image to the wavelet domain using Coiflets with three vanishing moments,

[2] Apply a threshold at two standard deviations, and

[3] Inverse-transformed the image to the signal domain

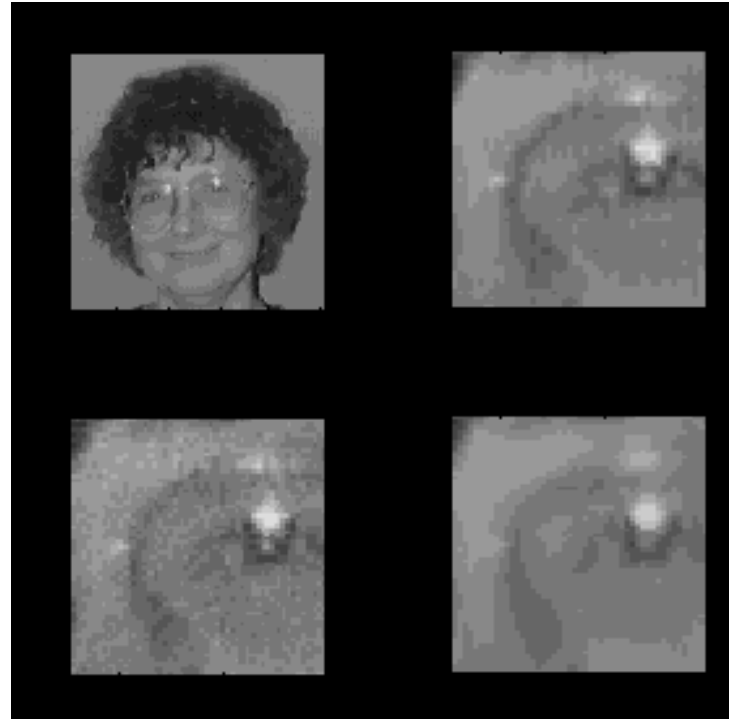

Fig. (A) Denoising an image of Ingrid Daubechies' left eye. The top left image is the original. At top right is a close-up image of her left eye. At bottom left is a close-up image with noise added. At bottom right is a close-up image, denoised

\section{IMPLEMENTATION}

Fig.3 shows the block diagram, for MRI denoising using Gaussian blurred intensity averaging method.We are taking Fig.2 for evaluating the performance of our algorithm.

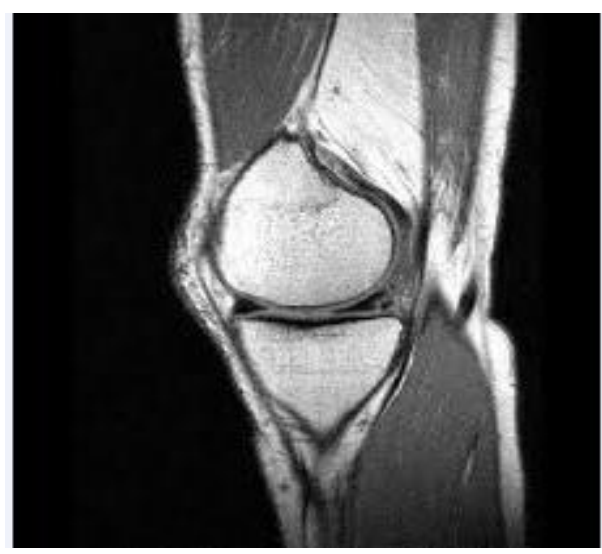

Fig.2 MRI of knee

We are averaging the intensity of pixels by finding forward and backward difference of intensity of neighboring pixels in $\mathrm{X}$ and $\mathrm{Y}$ direction. Then we will smooth an image by applying Gaussian blur and convolution. 


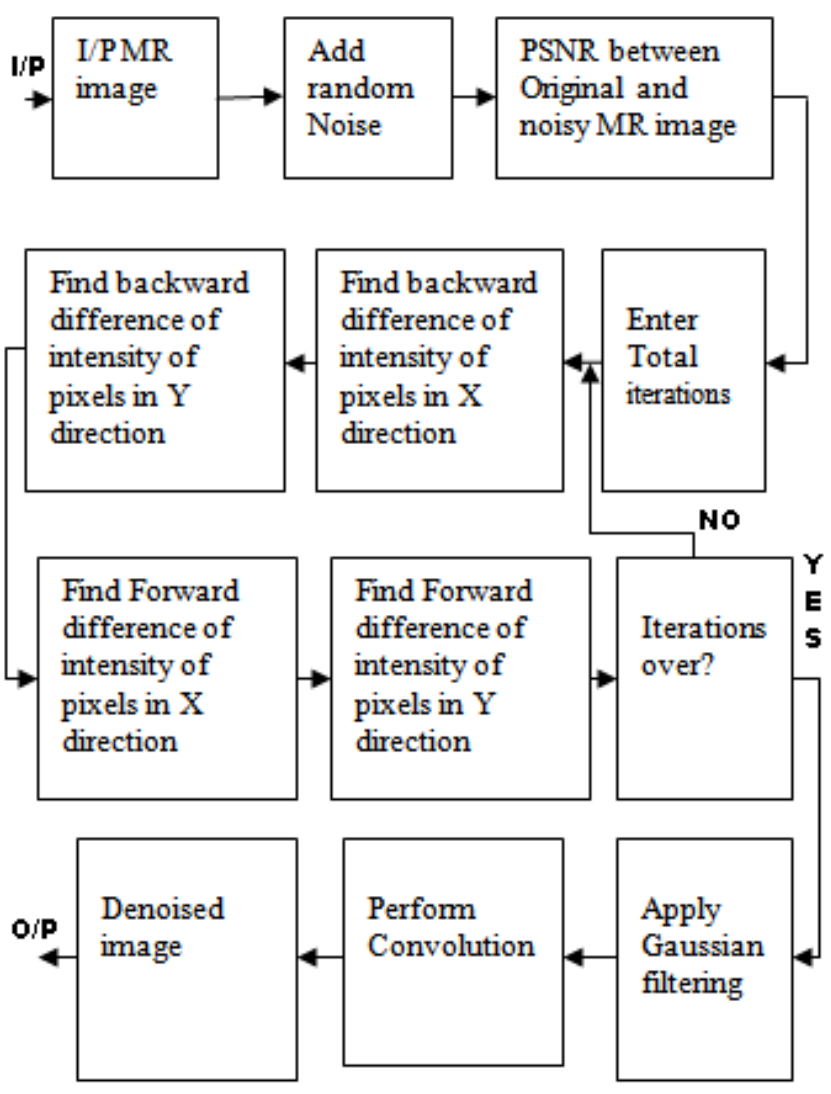

Fig.3 Block diagram of intensity averaging algorithm

\section{CONVOLUTION}

Noise removal can be performed by convolving the original image with the mask that represents law pass filter or by smoothing operation with Gaussian blur as discussed in section 4. convolution is a mathematical operation on two functions $\mathrm{f}$ and $\mathrm{g}$, producing a third function that is typically viewed as a modified version of one of the original functions, giving the area overlap between the two functions as a function of the amount that one of the original functions is translated. This convolution brings the value of each pixel into closer harmony with the values of its neighbors. In general, a smoothing filter sets each pixel to the average value, or a weighted average, of itself and its nearby neighbors; the Gaussian filter is just one possible set of weights.

Smoothing filters tend to blur an image, because pixel intensity values that are significantly higher or lower than the surrounding neighborhood would "smear" across the area. Because of this blurring, linear filters are seldom used in practice for noise reduction; they are, however, often used as the basis for nonlinear noise reduction filters.

\section{GAUSSIAN BLUR}

Gaussian blur, also known as Gaussian smoothing is used to blur (smooth) the image. Generally Gaussian blur reduces random noise from the image. Mathematically, Gaussian blur is equivalent to applying a convolution between image and Gaussian function [12, 13]. Gaussian distribution in 1-D is given as,
$G(x)=\frac{1}{\sqrt{2 \pi}} e^{-\frac{x^{2}}{2 \sigma^{2}}}$

Where $\sigma$ is the standard deviation of distribution. In 2-D, an isotropic Gaussian has the form,

$G(x, y)=\frac{1}{2 \pi \sigma^{2}} e^{-\frac{x^{2}+y^{2}}{2 \sigma^{2}}}$

Before we perform the convolution, we need to produce a discrete approximation of the Gaussian function as image is collection of pixels. Gaussian distribution is non-zero everywhere so ideally, we require an infinitely large convolution kernel but in practice we can truncate the kernel because Gaussian distribution is effectively zero more than about three standard deviations from the mean. The degree of smoothing depends on the value of standard deviation. The Gaussian outputs a 'weighted average' of each pixel's neighborhood, with the average weighted towards the value of the central pixels. So,

$\mathbf{I}(\mathbf{x}, \mathbf{y})=\mathbf{I}_{\mathbf{0}}(\mathbf{x}, \mathbf{y}) * \mathbf{G}(\mathbf{x}, \mathbf{y})$

$\mathrm{I}(\mathrm{X}, \mathrm{Y})=$ Gaussian blurred image, $\mathrm{I} 0(\mathrm{x}, \mathrm{y})=$ Noisy image

$\mathrm{G}(\mathrm{x}, \mathrm{y}, \mathrm{t})=$ Gaussian filter function

This is in contrast to the mean filter's uniformly weighted average.[14] Because of this, a Gaussian provides gentler smoothing and preserves edges better than a similarly sized mean filter. The mail problem with Gaussian filter is,

- Loss of fine detail

- Smoothing across boundaries

as shown in fig.3.

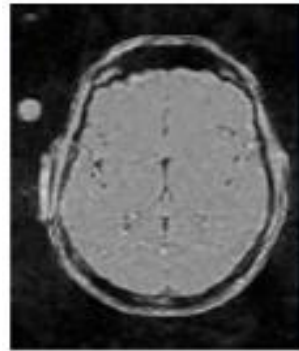

Fig. 4 (a)

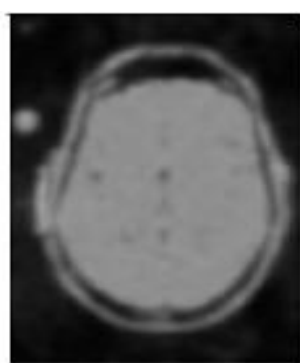

(b)
Fig. 4 (a) Before Gaussian blur (b) After Gaussian blur

This problem can be overcome by anisotropic diffusion [15, 16, and 17]

\section{ALGORITHM}

$>$ I/P Image.

$>$ Add Random noise

$>$ Find PSNR between Original and Noisy Image.

> Apply Magnitude Reconstruction using iteration method On MR Image

$>$ Enter iteration value. 
$>$ Find the backward difference of intensity of pixels in $\mathrm{X}$ direction till the iteration ends.

$>$ Find the backward difference of intensity of pixels in $\mathrm{Y}$ direction till the iteration ends

$>$ Find the forward difference of intensity of Pixel in $\mathrm{X}$ direction till the iteration ends.

$>$ Find the forward difference of intensity of Pixel in $Y$ direction till the iteration ends.

$>\quad$ Find PSNR between Original and Denoisy Image after iteration process [psnr2].

$>$ Apply Gaussian filters to blur the image.

$>$ Perform convolution.

$>$ Find PSNR between Original and Denoisyimage after Gaussian blurring. [psnr3].

$>$ Denoised Image

Now, we will evaluate the algorithm by taking different values of iterations (A).
I/P image

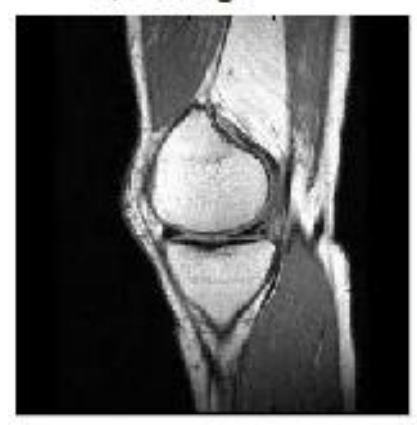

Fig.5 (a) Input image
Noisy Image

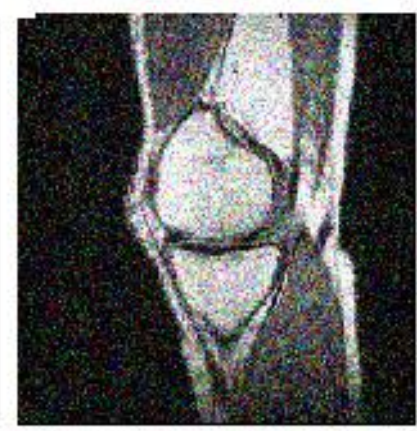

(b) Noisy input image
Now apply different values of "B" (no .of iterations) on noisy MR image as shown in fig.6 (b). As the value of "B" increases, we are getting more and more noise removal from noisy image as shown in fig. (6), (7) and (8). We are taking following parameters to evaluate the algorithm.

Psnr2:- PSNR between Original and Denoisy image after iteration process

psnr3:- PSNR between Original and Denoisy image after Gaussian blurring Image.

Mse2:-Difference between Original and Denoisy image after iteration process

Mse3:-Difference between Original and Denoisy image after Gaussian blurring Image.

\section{$\underline{\text { FOR B }=5}$}

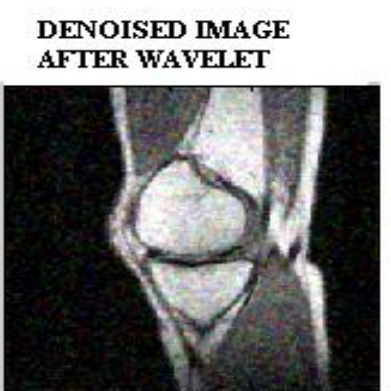

Fig.6 (a)

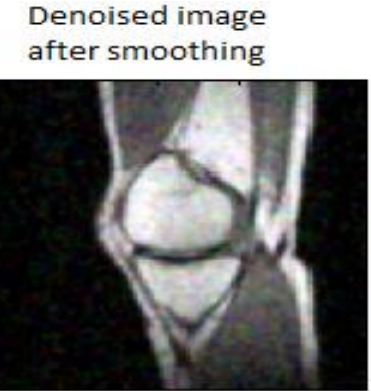

(b)
PSNR between original and denoisy image after iterations

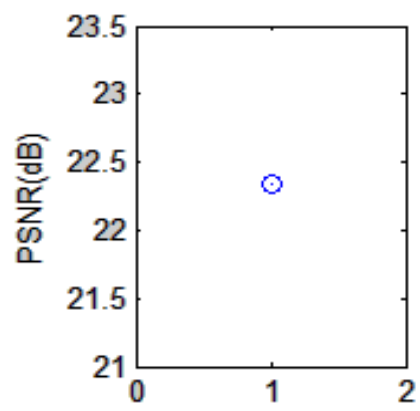

(c)
PSNR between original and denoisy image after smoothing

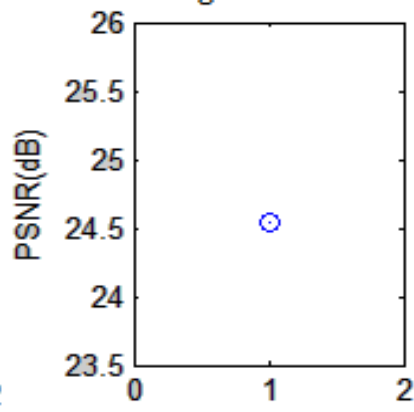

(d)

Fig.6 Denoised image after (a) iterations (b) Gaussian blur and convolution

Fig.6 PSNR between original and denoisy image after (c) iteration (d) Gaussian blur and convolution

Table-1

OBTAINED RESULT FOR B=5

\begin{tabular}{|l|l|}
\hline Psnr2=22.33 & Mse2=15.51 \\
\hline Psnr3=24.53 & Mse3=17.86 \\
\hline
\end{tabular}

\section{FOR B=10:-}

DENOISED IMAGE
AFTER WAVELET

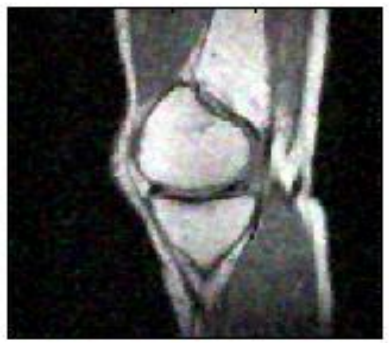

Fig.7 (a)
Denoised image after smoothing

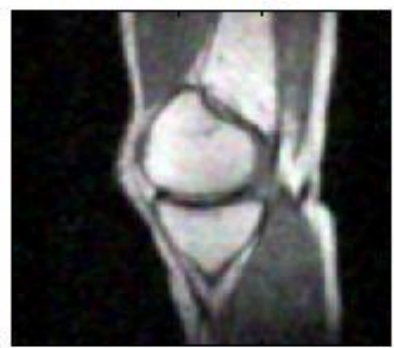

(b)
PSNR between original and denoisy image after iterations

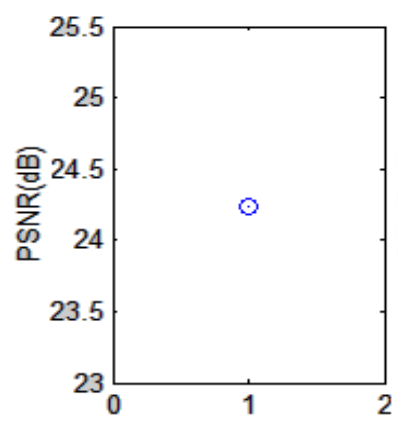

(c)
PSNR between original and denoisy image after smoothing

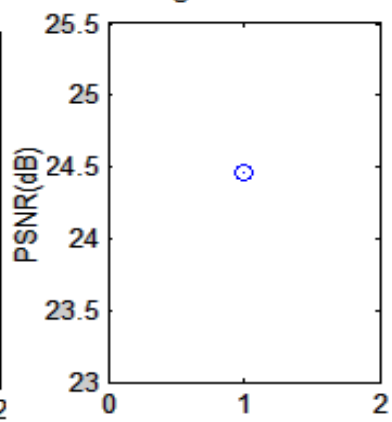

(d)

Fig.7 Denoised image after (a) iterations (b) Gaussian blur and convolution

Fig.7 PSNR between original and denoisy image after (c) iteration (d) Gaussian blur and convolution 
Table-2

OBTAINED RESULT FOR B=10

\begin{tabular}{|l|l|}
\hline Psnr2=23.10 & Mse2=16.63 \\
\hline Psnr3=28.24 & Mse3=18.06 \\
\hline
\end{tabular}

\section{FOR B=15:-}

\section{DENOISED IMAGE} AFTER WAVELET

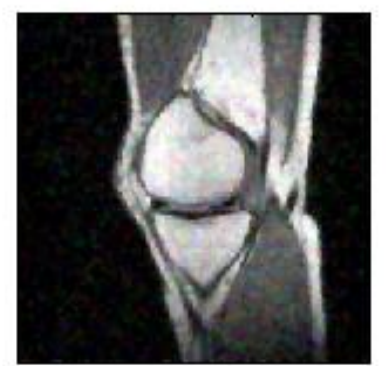

Fig.8(a)

PSNR between original and denoisy image after iterations

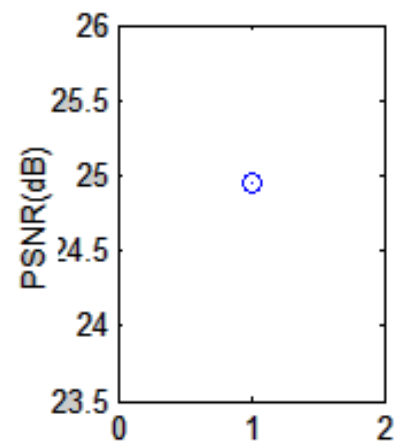

Denoised image after smoothing

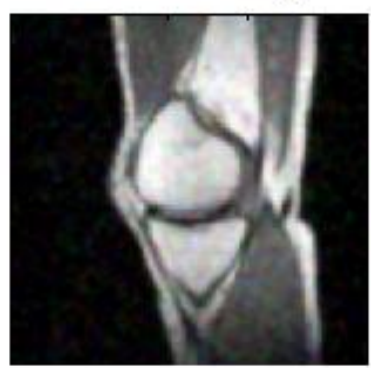

(b)

PSNR between original and denoisy image after smoothing

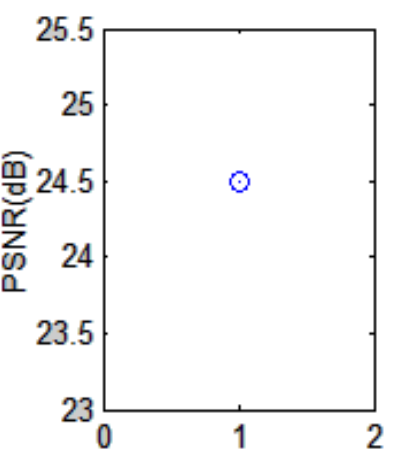

Fig.8 Denoised image after (a) iterations (b) Gaussian blur and convolution

Fig.8 PSNR between original and denoisy image after (c) iteration (d) Gaussian blur and convolution

Table-3

OBTAINED RESULT FOR A=20

\begin{tabular}{|l|l|}
\hline Psnr2=24.94 & Mse2=18.22 \\
\hline Psnr3=24.49 & Mse3=17.24 \\
\hline
\end{tabular}

\section{CONCLUSION}

From the above result we conclude that, our algorithm is efficient to remove the noise from MR image. Amount of noise removal from image depends on no. iterations (B). We can observe from above results that, as number of iterations increases, we are getting more and more noise removal from noisy MR image. Experimental results show that, we are getting good result in terms of PSNR and visuality.This algorithm is capable to remove noise from image and at the same time it is preserving fine details of image too. The problem with our algorithm is that we are only reconstructing the amplitude of noisy MR image and not phase. Denoisy image can be better if phase reconstruction is done. So future work is to implement phase reconstructing algorithm to improve the result. We also conclude that, for large value of iteration $(B>10)$, increment in PSNR is less compare to small values of iterations $(\mathrm{B}<10)$.

\section{REFERENCES}

[1] Isaac Bankman, "Handbook of Medical Imaging", Academic Press, 2000

[2] Mukesh C. Motwani, Mukesh C. Gadiya, Rakhi C.Motwani and Frederick C. Harris "Survey of Image Denoising Techniques"

[3] J.Rajeesh, R.S.Moni, S.Palanikumar \& T.Gopalakrishnan "Noise Reduction in Magnetic Resonance Images using Wave Atom Shrinkage" International Journal of Image Processing (IJIP), Volume(4) : Issue(2) 2010 pp: 131141.

[4] Milindkumar V. Sarode, Dr. Prashant R . Deshmukh "Performance Evaluation of Noise Reduction Algorithm in Magnetic Resonance Images "IJCSI International Journal of Computer Science Issues, Vol. 8, Issue 2, March 2011

[5] Milindkumar V. Sarode, Dr. Prashant R. Deshmukh "Performance Evaluation of Rician Noise Reduction Algorithm in Magnetic Resonance Images" Journal of Emerging Trends in Computing and Information Sciences Volume 2 Special Issue 2010-11 CIS Journal ISSN 2079- 8407 pp 39-44.

[6] YangWang and Haomin Zhou" Total VariationWaveletBased Medical Image Denoising" Hindawi Publishing CorporationInternational Journal of Biomedical Imaging Volume 2006, Article ID 89095

[7] S.Satheesh, Dr.KVSVR Prasad," Medical image denoising using adaptive threshold based on contourlet transform", Advanced Computing: An International Journal ( ACIJ ), Vol.2, No.2, March 2011

[8] José V. Manjón, Neil A. Thacker, Juan J. Lull, Gracian Garcia-Martí, Luís Martí- Bonmatí, Montserrat Robles "Multicomponent MR Image Denoising" Hindawi Publishing Corporation International Journal of Biomedical Imaging Volume 2009, Article ID 7 56897, 10 pages doi: $10.1155 / 2009 / 756897$

[9] Miguel E. Soto, Jorge E. Pezoa and Sergio N. Torres Thermal Noise Estimation and Removal in MRI: A NoiseCancellation Approach” 2011

[10] Marius Lysaker, Arvid Lundervold, and Xue-Cheng Tai " Noise Removal Using Fourth-Order Partial Differential Equation With Applications to Medical Magnetic Resonance Images in Space and Time" IEEE TransactionsOn Image Processing, VOL. 12, NO. 12, December 2003

[11] Dylan Tisdall and M. Stella Atkins "MRI denoising via phase error estimation" Proc. of SPIE Vol. 5747 (SPIE, Bellingham, WA, 2005) 16057422/05/\$15 - doi: 10.1117/12.595677. pp: 646-654

[12] Frederick M. Waltz and John W. V. Miller" An efficient algorithm for Gaussian blur using finitestate machines" SPIE Conf. on Machine Vision Systems for Inspection and Metrology VII, published in Boston, Nov. 1998 
[13] Gedraite, E.S.; Hadad, M ." Investigation on the effect of a Gaussian Blur in image filtering and segmentation" IEEE conference publication held at Zadar, ISSN- 1334-2630, Issue Date : 14-16 Sept. 2011

[14] Restrepo, A.; Bovik, A.C." Adaptive trimmed mean filters for image restoration'IEEE Journals and magazines, volume 36,Issue 8, ISSN : 0096-3518, Aug2002 .

[15] Qiang Wang; Yi Shen" The effects of fusion structures on image fusion performances", IEEE conference publication, Volume: 1,DOC:- 18-20 May 2004, ISSN: 1091-5281
[16] Pietro Perona and Jitendra Malik (July 1990 ). "Scale-space and edge detection using anisotropic diffusion". IEEE Transactions on Pattern Analysis and Machine Intelligence, 12 (7): 629-639. DOI:10.1109/34.56205.

[17] Yu-Li You; Wenyuan Xu; Tannenbaum, A.; Kaveh, M." Behavioral analysis of anisotropic diffusion in image processing" IEEE Journals, Volume: 5 ,Issue 11 ,DOI: 10.1109/83.541424, ISSN : 1057-7149

[18] B. Vidakovic and P. Muller, "Wavelets for Kids," 1994, unpublished. Part One, and Part Two. 